\title{
Nonequilibrium solid-solid phase transition in a lattice of liquid jets
}

\author{
Fabrizio Croccolo, ${ }^{1,2}$ Stefano Castellini, ${ }^{3}$ Frank Scheffold, ${ }^{1}$ and Alberto Vailati ${ }^{3, *}$ \\ ${ }^{1}$ Department of Physics, University of Fribourg, CH-1700 Fribourg, Switzerland \\ ${ }^{2}$ Laboratoire des Fluides Complexes et leurs Réservoirs - IPRA, UMR5150, E2S-Univ Pau \& Pays Adour/CNRS/Total, 64600 Anglet, France \\ ${ }^{3}$ Dipartimento di Fisica, Universitá degli Studi di Milano, I-20133 Milano, Italy
}

(Received 26 July 2018; published 11 December 2018)

\begin{abstract}
Solid-solid phase transitions are commonly encountered at the atomic scale in alloys and in superatomic mesoscopic systems of colloidal particles. Here we investigate a solid-solid phase transition occurring at the macroscopic scale between lattices of liquid jets with different symmetries generated by convection in a horizontal layer of a binary liquid mixture. In the absence of a shear stress, upwelling and downwelling jets arrange into two staggered square lattices with a spacing of approximately $3 \mathrm{~mm}$. Applying a shear stress triggers a phase separation of the square patterns into two centered-rectangular lattices drifting into opposite directions, each lattice being made either by upwelling or downwelling jets. This structural phase transition is reversible. The macroscopic nature of the system allows us to investigate the kinetics of the transition by direct visualization with shadowgraphy. The mechanism of the transition depends on the path followed. It occurs through a nucleation and growth mechanism when the shear stress is imposed, and through a martensitic transformation of the lattice when the stress is removed.
\end{abstract}

DOI: 10.1103/PhysRevE.98.063104

\section{INTRODUCTION}

Solid-solid phase transitions are widely investigated in atomic and colloidal systems, due to their relevance in natural phenomena such as the olivine-spinel transformation in Earth's mantle [1] and in technological processes [2,3], where they allow the development of devices based on the phase change in a single layer of atoms [4]. The two fundamental mechanisms involved in the transition are the diffusive motion of atoms and the martensitic transformation of the starting lattice into the final one. The same mechanisms determine the solid-solid transition in colloidal suspensions under the action of an external field [5,6]. A recent study on the solid-solid transition induced by an external field in ultrasoft colloids has shown that the selection of a martensitic or diffusive mechanism can depend on the path of the transformation [7]. The solid transition in colloidal suspension can also occur through a hybrid process, where the martensitic formation of a nucleus is followed by its diffusive growth [8]. Another mechanism for the transition, found both in colloidal [9] and atomic systems [10], proceeds by the formation of a transient melted phase separating two solid phases with different symmetries $[9,10]$. Solid-solid phase transitions can be partially understood by using Landau's theory of phase transitions. However, a consistent and general theoretical framework is not available to describe such transitions, because transitions between lattices lacking a group/subgroup relationship require dedicated phenomenological models $[11,12]$.

Ordered structures with various symmetry can be also obtained at the macroscopic scale during convection in fluid layers [13]. Under particular conditions, the convective flow

\footnotetext{
*alberto.vailati@unimi.it
}

arranges into streams of liquid of columnar shape, giving rise to the formation of jets crossing the liquid layer in the vertical direction [14-17], and arranges in a spoke pattern configuration that qualitatively mimics the arrangement of molecules in a two dimensional liquid [18]. Ordered square lattices of liquid jets can be obtained for convection in simple fluids and binary mixtures in the presence of poorly conductive boundaries, at Rayleigh numbers close to threshold [19-23]. The imposition of a shear stress, attained by inclining the layer of liquid to induce a large scale flow, significantly alters the convective patterns of a simple fluid [24-31]. In the case of a binary liquid mixture, recent experiments [32] and simulations [33] have shown that the imposition of marginal inclination induces a shear stress acting on the square lattice of jets, which determines the transition to an ordered configuration with different symmetry. This feature suggests the opportunity of using this kind of system as a model to investigate at the macroscopic scale the solid-solid phase transitions encountered in atomic and colloidal systems.

In this work we investigate nonequilibrium solid-solid phase transitions between lattices of convective jets generated by heating from below a binary liquid mixture with positive Soret coefficient. The jets act as the atoms of a two dimensional (2D) solid alloy due to bidimensional interactions at the macroscopic scale. A remarkable difference with atomic and colloidal systems is that jets can be created or annihilated at the boundaries of the system, the only constraint being that their average surface density is conserved. The main result of our work is that the solid-solid transition occurring at the macroscopic scale between square and centeredrectangular lattices of jets can occur either through a diffusive or a martensitic transformation, depending on the path chosen for the transition. The transition from a square to a centered-rectangular lattice is determined by the imposition 

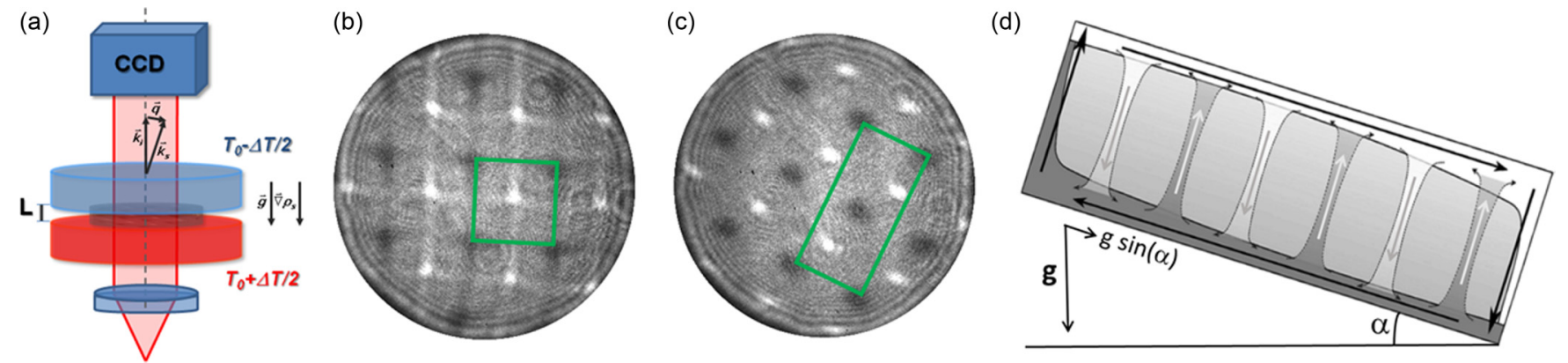

FIG. 1. Square and centered-rectangular lattices formed by liquid jets during convection in a binary liquid mixture. (a) Schematic setup of the shadowgraph optical diagnostics. The collimated light coming from a led source crosses the sample in the vertical direction and is deflected by the liquid jets, producing a shadow image of their configuration onto a charged coupled device sensor. The sample is sandwiched between a hot and a cold sapphire window whose temperature is set at $T_{0}+\Delta T / 2$ (bottom) and $T_{0}-\Delta T / 2$ (top). (b) Square lattice obtained with a temperature difference $\Delta T=8.0 \mathrm{~K}$, in the absence of inclination. Dark (bright) spots represent upwelling (downwelling) jets. (c) Centered-rectangular lattice obtained with a temperature difference $\Delta T=9.0 \mathrm{~K}$ and an inclination of $24 \mathrm{mrad}$. (d) Soret mass flow determines the formation of two unstable boundary layers at the top and bottom of the cell. Velocity fluctuations in the boundary layers are amplified by gravity and give rise to upwelling and downwelling jets crossing the sample in the vertical direction. The presence of an inclination of the sample gives rise to an in plane component $g \sin (\alpha)$ of the acceleration of gravity, which drives the large scale flow of the boundary layers (black arrows). The top boundary layer drifts downwards along the top boundary, and the bottom boundary layer drifts upwards along the bottom boundary.

of an anisotropic stress induced by a shear flow and involves the diffusive growth of a centered-rectangular pattern, which gradually invades the available space, starting from the formation of nuclei at the boundaries of the system. Conversely, the transition from a centered-rectangular to a square lattice is determined by the removal of the external stress and occurs through a martensitic transformation, where alternate rows of jets shift in opposite directions parallel to the direction of the stress to restore the square configuration.

\section{EXPERIMENTAL SYSTEM}

Jets are generated by applying a vertical temperature gradient, heating from below, to a horizontal layer of thickness $h=1.3 \mathrm{~mm}$ of the binary liquid mixture isobutylbenzene $/ n$ dodecane with a weight fraction concentration $c=50 \%$ [34]. When the mixture is heated from below, the unstable density profile generated by thermal dilation and by the concentration profile induced by the Soret effect can give rise to convective motions. Under particular conditions the convective flux takes the form of vertical jets of ascending and descending fluid, arranged into configurations mimicking the structure of amorphous [17] and crystalline alloys [21,32,33]. When the layer of liquid is horizontal, square lattices of jets can be obtained both for heat convection in simple liquids [20] and for solutal convection in binary mixtures [21], provided that the sample is close to the threshold of convection and that the horizontal boundaries are poor conductors of heat and mass, respectively. Theoretical models developed for simple fluid show that in the presence of insulating boundaries a square pattern of jets represents the stable configuration that maximizes the transfer of heat [19]. The conditions leading to the formation of square patterns in mixtures are more complicated, because the presence of the Soret effect determines an additional contribution to the density profile of the binary mixture, beyond that generated by the thermal dilation of the sample. In the case of a binary mixture with positive Soret coefficient, both the temperature and concentration gradient contribute to the destabilization of the liquid layer and stable square solutions are found close to the threshold for RayleighBénard convection [22,23].

The condition leading to the development of convective jets can be parametrized by the dimensionless Rayleigh number $\mathrm{Ra}=g \Delta \rho_{T} h^{3} /\left(\eta D_{T}\right)$ and by the Prandtl numer $\operatorname{Pr}=v / D_{T}$, where $g$ is the acceleration of gravity, $\Delta \rho_{T}$ is the density difference generated by thermal dilation of the sample, $h$ is the thickness of the liquid layer, $\eta$ is the shear viscosity, $D_{T}$ is the thermal diffusivity, and $v$ is the kinematic viscosity. In the case of a horizontal layer of a binary liquid mixture the imposition of a temperature gradient induces a nonequilibrium Soret mass flux proportional to the applied temperature gradient $j=-\rho D S_{T} c(1-c) \nabla T$. Here $S_{T}$ is the Soret coefficient. As a consequence, a solutal density difference $\Delta \rho_{c}=S_{T} c(1-c) \Delta T$ is generated inside the sample [35]. Stable convective jets arranged into ordered lattices develop at Rayleigh numbers of the order of 1400 [32].

Shadowgraph images of the jets appear as dark and bright spots [Figs. 1(b) and 1(c)] distributed across the plane. Bright spots correspond to downwelling jets and dark spots to upwelling ones. The development of jets is triggered by the formation of unstable solutal boundary layers (BLs) close to the upper and lower sapphire windows. The growth of the BLs is determined by the Soret mass flow. At the beginning, the thickness of the BLs increases uniformly in time. When the boundary layers reach a critical thickness they become unstable against fluctuations and convection sets in [36-38]. Imposing a small inclination to the horizontal layer of the binary mixture determines formation of a large scale flow inside the liquid sample, and the development of a shear stress on the crystalline structures formed by the jets. The strength of the large scale flow depends on the inclination angle, a feature which allows us to control accurately the shear stress imposed to the system of jets. By tuning the temperature gradient and by changing the inclination of the cell the system can be 

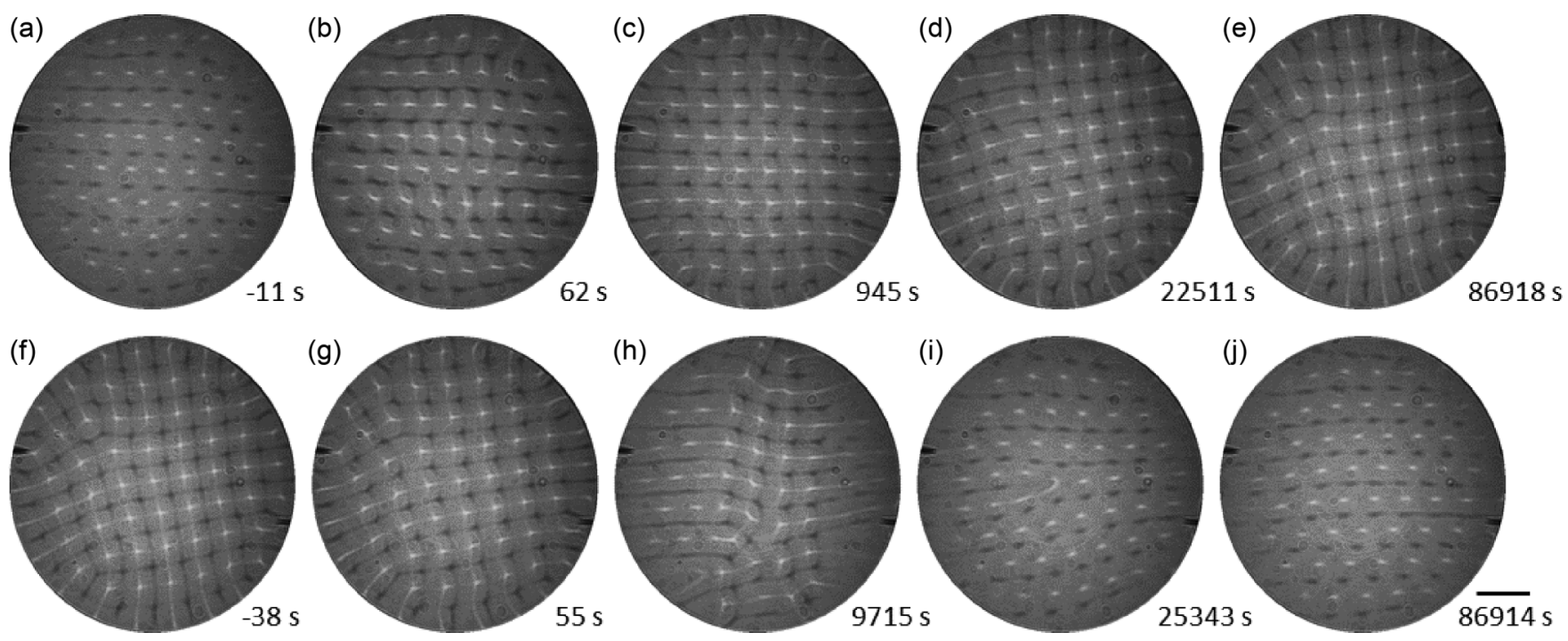

FIG. 2. Time evolution of the transition between the square and centered-rectangular lattice. (a)-(e) Transition from centered-rectangular to square lattices (Supplemental Material movie 1 [39]). (f)-(j) Transition from square to centered-rectangular lattices (Supplemental Material movie 2 [39]). Time $t=0$ corresponds to the instant where an inclination of $24 \mathrm{mrad}$ was either removed or imposed from the layer of liquid to trigger a shear stress. A stationary temperature difference of $10.1 \mathrm{~K}$ was applied to the sample during the measurement. Scale bar: $5 \mathrm{~mm}$.

brought into stationary states where jets are arranged either in a square lattice [Fig. 1(b)] or in a centered-rectangular lattice [Fig. 1(c)]. The square and centered-rectangular structures can be accessed independently by preparing the system in an initial configuration where the concentration of the mixture is uniform and suddenly applying a carefully chosen temperature difference [32,33].

In the absence of inclination, the jets are arranged in a structure formed by two staggered square patterns, where ascending and descending jets are as far as possible from each other. In the presence of an inclination as small as a few milliradians, the jets arrange into two staggered centeredrectangular lattices drifting into opposite directions [Fig. 1(d) and Supplemental Material movies [39]] [32]. Under this condition, the inclination of the layer of liquid determines the presence of a flow parallel to the boundaries: downwelling jets move in the direction of the residual gravity, while the upwelling ones move in the opposite direction. The distribution of jets undergoes a rearrangement consisting in the formation of parallel alternate lanes of jets flowing into opposite directions, in a "superhighway convection" (SHC) [32]. This lane formation phenomenon occurs quite generally in complex systems in the presence of counterflows. For example, this phenomenon has been reported in the case of pedestrians crossing a lane from opposite directions [40].

\section{RESULTS AND DISCUSSION}

The fact that the system of jets can access two ordered phases with different symmetry raises the following fundamental questions: can a transition between the square and centered-rectangular pattern be obtained by preparing the system in one of the two states and by applying or removing a shear stress? If such a transition exists, does it occur through a diffusive or a martensitic transformation? What is the relation between the primitive vectors of the square and centered-rectangular Bravais lattices involved in the transition and how do these vectors depend on the imposed shear stress?

To investigate the transition between the square and centered rectangular lattice we have prepared the system in the centered-rectangular phase and triggered the transition back and forth by changing the inclination of the sample (Fig. 2, Supplemental Material movies 1 and 2 [39]). The initial configuration has been prepared by tilting the setup at an angle of $36 \mathrm{mrad}$ and by imposing a 10.1-K temperature difference. After waiting a suitable time [32] a stationary configuration with two centered-rectangular lattices drifting into opposite directions has been obtained [Fig. 2(a)]. In this configuration the jets of each species are organized into horizontal lanes aligned with the direction of motion. The removal of the inclination leads to the rapid formation of an oblique lattice $(t=62 \mathrm{~s})$. The jets then align also along the direction perpendicular to the lanes [Figs. 2(b)-2(d)] and eventually form a structure made by two staggered square lattices [Fig. 2(e)]. The transition from the centered-rectangular to the square lattice [Figs. 2(a)-2(e)] is achieved through a martensitic transformation of the original lattice, in the absence of an intermediate liquid state. The transition between the centeredrectangular and square lattices is reversible. Starting from square lattices [Fig. 2(f)], the imposition of the inclination gives rise to a small shift of the bright and dark jets into opposite directions parallel to the inclination [Fig. 2(g)], followed by the development of two centered-rectangular lattices at the sides of the observation window, which slowly grow through a nucleation and growth mechanism [Fig. 2(h)]. These two centered-rectangular phases coexist for some time with the square one, which occupies a band at the center of the cell [Fig. 2(h)]. The transition from the square to the centered-rectangular lattice involves the formation of an interface between the two lattices. The presence of defects at the interface allows the coexistence of the two phases with different symmetry and the diffusive growth of the 
(a)

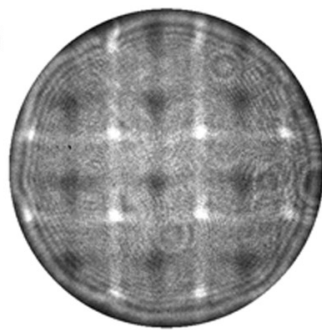

0 mrad $\mathrm{Ra}=1240$

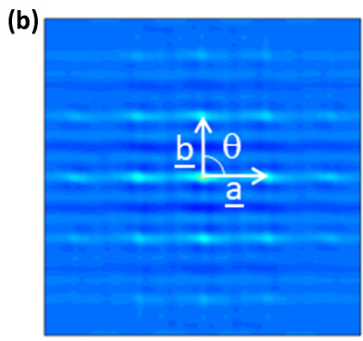

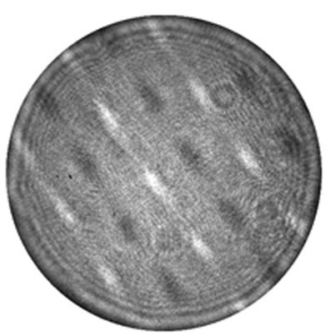

6 mrad $\mathrm{Ra}=1240$

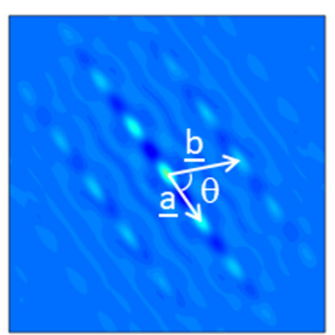

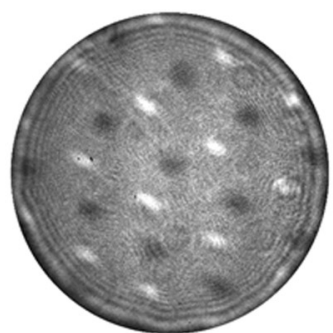

$18 \mathrm{mrad} \mathrm{Ra}=1370$

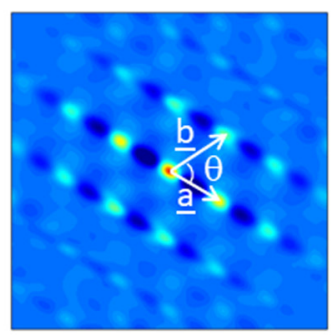

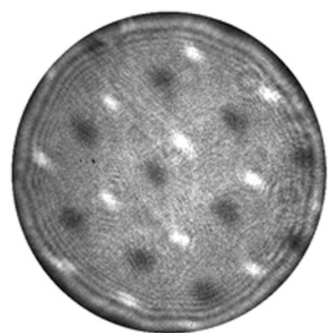

$24 \mathrm{mrad} R \mathrm{Ra}=1400$

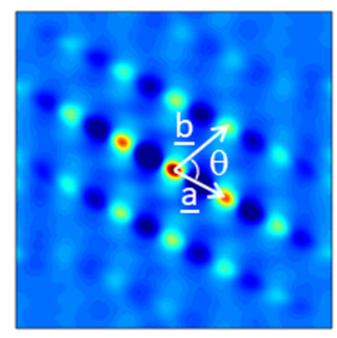

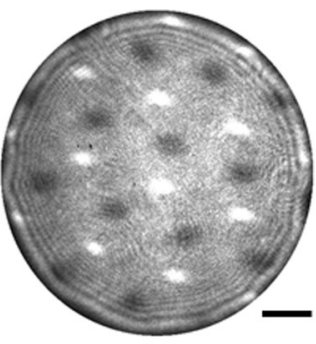

36 mrad $\mathrm{Ra}=1410$

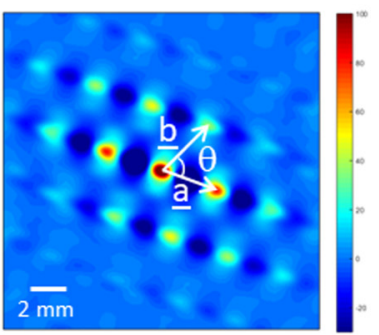

(c)

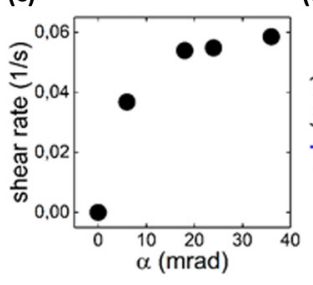

(d)

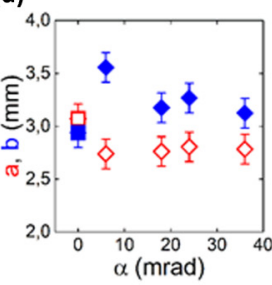

(e)

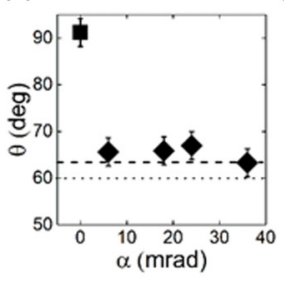

(f)

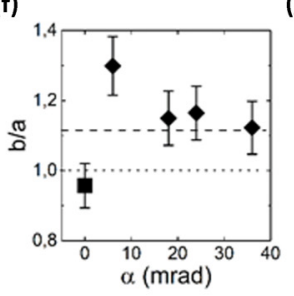

(g)

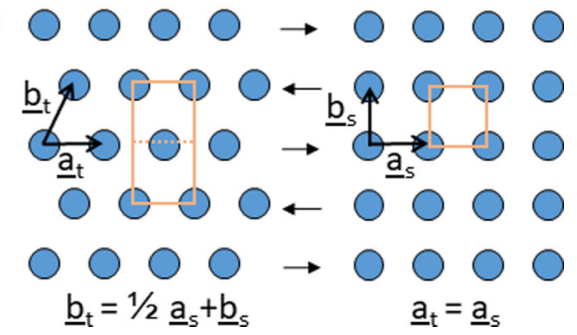

FIG. 3. Relation between the primitive lattice vectors of the square and centered rectangular lattices. (a) Stationary lattices obtained under different shear stress conditions. In the presence of inclination, patterns align in the direction of shear. Scale bar: $2 \mathrm{~mm}$. (b) Spatial autocorrelation functions obtained under the conditions shown in (a), averaged over sequences of 400 images recorded at a frame rate of one image per $30 \mathrm{~s}$. $\underline{a}$ and $\underline{\mathrm{b}}$ represent the primitive vectors and $\theta$ the angle between them. (c) Shear rate plotted as a function of the inclination angle $\alpha$. (d) Moduli of the primitive vectors a (hollow) and $\mathrm{b}$ (full), (e) angle between the primitive vectors, and (f) ratio $b / a$ plotted as a function of the inclination angle $\alpha$ for the square (squares) and centered rectangular (diamonds) lattices. The dotted lines in (e) and (f) show the predictions for a triangular phase, while the dashed lines correspond to the predictions for a centered-rectangular one. (g) Martensitic transformation from a centered-rectangular to a square lattice, where alternate rows of jets move coherently into opposite directions to form the new phase. The rectangular unit cell of the centered-rectangular lattice is twice as large as the square unit cell of the square lattice.

centered-rectangular phase. Some of these defects can still survive after the square pattern has disappeared [center of Fig. 2(i)], but fade eventually, thus forming a defect-free centered-rectangular lattice [Fig. 2(j)].

In order to investigate the relation between the typical length scales of the square and centered-rectangular lattices we performed a systematic characterization of the primitive lattice vectors as a function of the shear stress imposed to the system, which can be varied accurately by changing the inclination angle of the cell with respect to the horizontal plane. Starting from the condition where the concentration of the sample is uniform, we have created stable lattices of jets under different shear stress conditions [Fig. 3(a)]. While the orientation of the square pattern is random, the orientation of patterns generated in the presence of inclination corresponds to the direction of shear. To achieve a statistical characterization of the primitive lattice vectors we have determined the 2D spatial autocorrelation function of shadow images for each configuration shown in Fig. 3(a). The autocorrelation functions have been averaged over sequences of 400 shadow images taken every $30 \mathrm{~s}$ at fixed inclination [Fig. 3(b)]. The drift velocities $v_{b}$ and $v_{w}$ of the black and white lattices, determined by tracking the position of the jets, allow us to estimate the shear rate $\dot{\gamma}=\left(v_{b}-v_{w}\right) / h$ [Fig. 3(c)]. The in plane component of the acceleration of gravity in the presence of inclination varies in the range $0<g \sin (\alpha)<0.035 \mathrm{~m} / \mathrm{s}^{2}$ and the shear rate in the range $0<\dot{\gamma}<5.9 \times 10^{-2} \mathrm{~s}^{-1}$. The primitive lattice vectors have been determined from the position of the nearest neighbors peaks with respect to the central peak [Fig. 3(b)]. Their moduli are nearly identical in the case of the square lattice, but differ significantly for the centered-square lattice [Fig. 3(d)]. This is a first indication that the centered-rectangular lattice is not a triangular lattice. Further evidence comes from the measurement of the angle between the primitive vectors, and from the ratio $b / a$. The measured angle is compatible with a value $\theta=63.4^{\circ}$ [Fig. 3(e)], while the ratio with a value $b / a=\sqrt{5} / 2$ [Fig. 3(f)]. These two features show that the centered-rectangular lattice is made by rectangular unit cells with aspect ratio equal to 2 [Fig. $3(\mathrm{~g})]$. This configuration is compatible with the martensitic transition from the centeredrectangular to the square lattice shown in Figs. 2(a)-2(e) and Supplemental Material movie 1 [39], where the imposition of a shear stress determines a relative movement of $a / 4$ of the 
rows of each square lattice [Fig. 3(g)]. A striking feature of the phase transitions in the lattice of liquid jets is that they occur sharply as a function of the inclination angle. This is apparent from Figs. 3(d)-3(f), which show that in the presence of a tilt angle as small as $6 \mathrm{mrad}$ the centered-rectangular pattern has already the same features of those present at much larger tilt angles. The experimental results currently do not allow us to establish whether the transitions occur for inclination angles larger than a critical threshold, or without any threshold. A more refined investigation of this aspect would require performing challenging experiments by using smaller shear rates at tiny inclination angles of the order of 2-3 mrad.

A deeper understanding of the kinetics of the transition would require the development of simulations of the hydrodynamics of the system where the inclination of the liquid layer can be changed continuously, as it occurs in experiments. The formation of square patterns is currently well understood from the theoretical point of view. Busse and Riahi have shown that in the presence of poorly conductive boundaries the square configuration represents the unstable solution leading to the maximum heat transfer [19].

Conversely, the pattern formation process in an inclined layer of a binary liquid mixture has been investigated only recently using computational methods by Alonso et al. [33]. The authors of this study simulated the three dimensional Boussinesq equations for a binary liquid mixture with positive Soret coefficient, using parameters mirroring those of experiments on SHC [32] and solving numerically the equations by using the algorithms described in [41]. These simulations show that the Soret effect determines a vertical stratification of the concentration of the mixture. An important result is that the large scale flow stratifies the concentration of the mixture along the direction of inclination by advecting concentration along the direction of inclination, thus determining the creation of stable concentration gradient along the horizontal direction. The large scale flow of the fluid is apparent from the mapping of the velocity field, which shows that the circulation occurs mostly downhill in the top half of the fluid layer, and uphill in the bottom half. Interestingly, at the mid-height of the cell, where the large scale velocity vanishes, the flow organizes into parallel lanes. The mapping of the temperature field shows the presence of hot and cold spots arranged into parallel lanes, corresponding to the upwelling or downwelling jets shown in Figs. 1-3. The motion of these hot spots corresponds to periodic solutions of the Navier-Stokes equations. Remarkably, the numerical solutions are in excellent agreement with the results of experiments on SHC [32], thus confirming the effectiveness of the numerical methods used to solve the Boussinesq Navier-Stokes equations in the presence of the Soret effect.

However, so far an accurate experimental investigation of the typical length scales of the SHC pattern has not been performed. One of the aims of our work is to fill this gap and provide robust experimental results for the structure of the square and SHC patterns, which will provide an important reference point for further theoretical and simulation work. In this respect, a remarkable result of our experiments is that the square and centered-rectangular lattices involve a single common length scale $\Lambda$, irrespective of the inclination angle, and this length scale is preserved after the transition. In the case of the square lattice the length scale corresponds to the side of each square. In the case of the centered rectangular lattice $\Lambda$ determines both the distance between jets aligned in a row, and the distance between adjacent rows of upwelling or downwelling jets. As shown in Fig. $3(\mathrm{~d}), \Lambda \approx 2.75 \mathrm{~mm}$ is about twice as large as the sample thickness $h=1.3 \mathrm{~mm}$. This suggests that the length scales of the square and SHC patterns are completely determined by the thickness of the layer. A more stringent check would require performing experiments with different layer thicknesses. Experiments performed by directly triggering the instability in the presence or absence of inclination (Fig. 3) or by first triggering the instability and then changing the inclination of the liquid layer (Fig. 2) show that the pattern formation process is not affected by the initial conditions and depends only on the Rayleigh number and inclination angle. There is then no evidence of the influence of the initial state, the choice of the pattern being driven by the periodic solutions of the Navier-Stokes equations as evidenced by numerical simulations.

In conclusion, although the lattice of jets that we have investigated is intrinsically a nonequilibrium system, the path dependent mechanism of the transition between the square and centered-rectangular structures is akin to that reported for the solid-solid transition observed in a system of ultrasoft colloids by imposing an external field [7]. In the case of ultrasoft colloids, the imposition of an electric field determines a diffusive transition from a face-centered-cubic lattice into a body-centered-tetragonal lattice through the nucleation and growth of domains. The removal of the external field determines a martensitic transformation of the tetragonal lattice into a metastable body-centered-orthorhombic lattice, which relaxes to the original face-centered-cubic lattice when annealed. In the case of our macroscopic system of jets, the imposition of a shear stress determines a diffusive transition from a square lattice to a centered-rectangular one, while the removal of the stress determines a martensitic transition of the centered-rectangular structure to the square one. This result indicates that path dependent mechanisms for solidsolid phase transitions are present in a wide variety of systems, even at macroscopic length scales.

\section{METHODS}

The sample is a binary liquid mixture of isobutylbenzene $/ n$-dodecane of concentration $c=50 \% \mathrm{w} / \mathrm{w}$. Its thermophysical properties are [34] as follows: separation ratio $\psi=\Delta \rho_{c} / \Delta \rho_{T}=0.13$, diffusion coefficient $D=$ $9.5 \times 10^{-10} \mathrm{~m}^{2} \mathrm{~s}^{-1}$, Soret coefficient $S_{T}=3.9 \times 10^{-3} \mathrm{~K}^{-1}$, $d n / d T=-4.5 \times 10^{-4} \mathrm{~K}^{-1}, d n / d c=6.3 \times 10^{-2}$, thermal expansion coefficient $\alpha=9.9 \times 10^{-5} \mathrm{~K}^{-1}$, solutal expansion coefficient $\beta=0.13$, mass density $\rho=0.7925 \mathrm{~g} \mathrm{~cm}^{-3}$, shear viscosity $\eta=1.1 \times 10^{-2} \mathrm{P}$.

Two different thermal gradient cells sharing the same design concept were used to visualize the jets [42-44]. In both cells the sample is delimited by two sapphire windows kept at a distance $h=1.30 \mathrm{~mm}$ and is sealed by a circular Viton O-ring gasket with an inner radius $R=13 \mathrm{~mm}$. This geometry corresponds to a moderately high aspect ratio $R / h=10$ of the sample. The sapphire windows are in contact with two annular thermoelectric devices connected to two independent 
proportional integral derivative temperature controllers. The temperature of each window can be controlled independently with an absolute accuracy of $0.01 \mathrm{~K}$ and a relative rms stability of $0.001 \mathrm{~K}$ over $24 \mathrm{~h}$. The performances and reliability of the cells have been established during several series of experiments on devices sharing a similar conceptual design $[17,36,42,43]$. One cell (cell A) has an optical clear aperture with a diameter $\phi_{A}=26 \mathrm{~mm}$, which guarantees a complete visualization of the sample including its boundaries and the observation of a large number of jets [44]. The other cell (cell B) has a smaller clear aperture $\phi_{B}=13 \mathrm{~mm}$, with the advantage of a more uniform thermalization of the sample, reflected by a more regular configuration of the structures formed by liquid jets [32].

The jets are visualized by using the shadowgraph projection technique $[45,46]$. The light source is a superluminous diode (Superlum Diodes, Broad Lighter S680) with a wavelength of $683 \mathrm{~nm}$, coupled to a single mode optical fiber. The diverging beam out of the fiber is collimated by using an achromatic doublet lens. In the case of cell B, no other lens is used after the sample cell and a charged coupled device Vosskühler CCD4000 detector with a resolution of $2048 \times 2048$ pixels is placed at a distance of $26 \mathrm{~cm}$ from the sample. Cell A can be tilted by using a calibrated screw, which allows us to impose inclination angles with an accuracy better than $2 \mathrm{mrad}$. In the case of cell $\mathrm{B}$ an achromatic doublet is used to conjugate a plane located at a distance of $26 \mathrm{~cm}$ from the cell to sensor plane of a JAI CV M300 camera with a resolution of $768 \times 576$ pixel. The shadowgraph diagnostics and the sample cell are fixed to a vertical optical bench which can be easily tilted by using calibrated screws while running a measurement. Before starting measurements in the presence of inclination, we calibrated the reference horizontal plane by leveling it with a precision bubble level. Subsequently, we performed test measurements in the absence of inclination, lasting as much as one month, to check for the absence of drift motions. These measurements confirmed the accuracy of the horizontal leveling.

\section{ACKNOWLEDGMENTS}

The authors acknowledge discussion with G. Onida and D. Pini. This project has benefited from support from the Swiss National Science Foundation via Project No. 169074 and the National Center of Competence in Research Bio-Inspired Materials. A.V. acknowledges support from Transition Grant No. 2014 of the University of Milan. F.C. acknowledges financial support from the European Union under FP7 PEOPLE Marie Curie Intra European Fellowship, Contract No. IEF-251131, DyNeFI Project.
[1] P. C. Burnley and H. W. Green II, Stress dependence of the mechanism of the olivine-spinel transformation, Nature (London) 338, 753 (1989).

[2] Y. Wang et al., Structural phase transition in monolayer $\mathrm{MoTe}_{2}$ driven by electrostatic doping, Nature (London) 550, 487 (2017).

[3] Y.-C. Lin, D. O. Dumcenco, Y.-S. Huang, and K. Suenaga, Atomic mechanism of the semiconducting-to-metallic phase transition in single-layered $\mathrm{MoS}_{2}$, Nat. Nanotechnol. 9, 391 (2014).

[4] B. Radisavljevic, A. Radenovic, J. Brivio, V. Giacometti, and A. Kis, Single-layer $\mathrm{MoS}_{2}$ transistor, Nat. Nanotechnol. 6, 147 (2011).

[5] J. A. Weiss, D. W. Oxtoby, D. G. Grier, and C. A. Murray, Martensitic transition in a confined suspension, J. Chem. Phys. 103, 1180 (1995).

[6] A. Yethiraj, A. Wouterse, B. Groh, and A. van Blaaderen, Nature of an Electric-Field-Induced Colloidal Martensitic Transition, Phys. Rev. Lett. 92, 058301 (2004).

[7] P. S. Mohanty, P. Bagheri, S. Nöjd, A. Yethiraj, and P. Schurtenberger, Multiple Path-Depedent Routes for Phase-Transition Kinetics in Thermoresponsive and Field-Responsive Ultrasoft Colloids, Phys. Rev. X 5, 011030 (2015).

[8] Y. Peng, W. Li, F. Wang, T. Still, A. G. Yodh, and Y. Han, Diffusive and martensitic nucleation kinetics in solidsolid transitions of colloidal crystals, Nat. Commun. 8, 14978 (2017).

[9] Y. Peng, F. Wang, Z. Wang, A. M. Alsayed, Z. Zhang, A. G. Yodh, and Y. Han, Two-step nucleation mechanism in solidsolid phase transitions, Nat. Mater. 14, 101 (2014).
[10] S. Pogatscher, D. Leutenegger, J. E. K. Schawe, P. J. Uggowitzer, and J. F. Löffler, Solid-solid phase transitions via melting in metals, Nat. Commun. 7, 11113 (2016).

[11] P. Toledano and V. Dmitriev, Reconstructive Phase Transitions (World Scientific, Singapore, 1996).

[12] D. M. Hatch, T. Lookman, A. Saxena, and H. T. Stokes, Systematics of group-nonsubgroup transitions: Square to triangle transition, Phys. Rev. B 64, 060104(R) (2001).

[13] M. C. Cross and P. C. Hohenberg, Rev. Mod. Phys. 65, 851 (1993).

[14] F. H. Busse and J. A. Whitehead, Oscillatory and collective instabilities in large Prandtl number convection, J. Fluid Mech. 66, 67 (1974).

[15] D. P. McKenzie and F. Richter, Convection currents in the earth's mantle, Sci. Am. 235, 72 (1976)

[16] A. La Porta and C. M. Surko, Convective Instability in a Fluid Mixture Heated from Above, Phys. Rev. Lett. 80, 3759 (1998)

[17] S. Mazzoni, F. Giavazzi, R. Cerbino, M. Giglio, and A. Vailati, Mutual Voronoi Tessellation in Spoke Pattern Convection, Phys. Rev. Lett. 100, 188104 (2008).

[18] F. Dubuffet and D. A. Yuen, A thick pipe-like heat-transfer mechanism in the Mantle: Nonlinear Coupling Between 3-D Convection and Variable Thermal Conductivity, Geophys. Res. Lett. 27, 17 (2000).

[19] F. H. Busse and N. Riahi, Nonlinear convection in a layer with nearly insulating boudaries, J. Fluid. Mech. 96, 243 (1980).

[20] P. Le Gal and V. Croquette, Appearance of a square pattern in a Rayleigh-Benard experiment, Phys. Fluids 31, 3440 (1988).

[21] E. Moses and V. Steinberg, Competing Patterns in a Convective Binary Mixture, Phys. Rev. Lett. 57, 2018 (1986). 
[22] B. Huke and M. Lücke, Roll, square, and cross-roll convection in ferrofluids, J. Magn. Magn. Mater. 289, 264 (2005).

[23] S. Weggler, B. Huke, and M. Lücke, Roll and square convection in binary liquids: A few-mode Galerkin model, Phys. Rev. E 81, 016309 (2010).

[24] R. M. Clever and F. H. Busse, Instabilities of longitudinal convection rolls in an inclined layer, J. Fluid. Mech. 81, 107 (1977).

[25] F. H. Busse and R. M. Clever, Three-dimensional convection in an inclined layer heated from below, J. Eng. Math. 26, 1 (1992).

[26] F. H. Busse and R. M. Clever, Bursts in inclined layer convection, Phys. Fluids 12, 2137 (2000).

[27] K. E. Daniels, B. B. Plapp, and E. Bodenschatz, Pattern Formation in Inclined Layer Convection, Phys. Rev. Lett. 84, 5320 (2000).

[28] K. E. Daniels and E. Bodenschatz, Defect Turbulence in Inclined Layer Convection, Phys. Rev. Lett. 88, 034501 (2002).

[29] G. Seiden, S. Weiss, J. H. McCoy, W. Pesch, and E. Bodenschatz, Pattern Forming System in the Presence of Different Symmetry-Breaking Mechanisms, Phys. Rev. Lett. 101, 214503 (2008).

[30] S. Weiss, G. Seiden, and E. Bodenschatz, Pattern formation in spatially forced thermal convection, New J. Phys. 14, 053010 (2012).

[31] A. Zebib and M. M. Bou-Ali, Inclined layer Soret instabilities, Phys. Rev. E 79, 056305 (2009).

[32] F. Croccolo, F. Scheffold, and A. Vailati, Effect of a Marginal Inclination on Pattern Formation in a Binary Liquid Mixture Under Thermal Stress, Phys. Rev. Lett. 111, 014502 (2013).

[33] A. Alonso, I. Mercader, and O. Batiste, Time-dependent patterns in quasivertical cylindrical binary convection, Phys. Rev. E 97, 023108 (2018).

[34] J. K. Platten, M. M. Bou-Ali, P. J. Costesèque, F. Dutrieux, W. Köhler, C. Leppla, S. Wiegand, and G. Wittko, Benchmark values for the Soret, thermal diffusion and diffusion coefficients of three binary organic liquid mixtures, Philos. Mag. 83, 1965 (2003).
[35] S. R. de Groot and P. Mazur, Nonequilibrium Thermodynamics (North-Holland, Amsterdam, 1962).

[36] R. Cerbino, S. Mazzoni, A. Vailati, and M. Giglio, Scaling Behavior for the Onset of Convection in a Colloidal Suspension, Phys. Rev. Lett. 94, 064501 (2005).

[37] F. Giavazzi and A. Vailati, Scaling of the spatial power spectrum of excitations at the onset of solutal convection in a nanofluid far from equilibrium, Phys. Rev. E 80, 015303(R) (2009).

[38] S. Messlinger, C. Kramer, J. J. Schmied, F. Winkel, W. Schöpf, and I. Rehberg, Experimental observations of Soret-driven convection in the transient diffusive boundary layer, Phys. Rev. E 88, 053019 (2013).

[39] See Supplemental Material at http://link.aps.org/supplemental/ 10.1103/PhysRevE.98.063104 for videos of the solid-solid transition.

[40] M. Moussaïd, D. Helbing, and G. Theraulaz, How simple rules determine pedestrian behavior and crowd disasters, Proc. Natl. Acad. Sci. USA 108, 6884 (2011).

[41] I. Mercader, O. Batiste, and A. Alonso, An efficient spectral code for incompresible flows in cylindrical geometries, Comput. Fluids 39, 215 (2010).

[42] M. Bernardin, F. Comitani, and A. Vailati, Tunable heat transfer with smart nanofluids, Phys. Rev. E 85, 066321 (2012).

[43] F. Croccolo, H. Bataller, and F. Scheffold, A light scattering study of non equilibrium fluctuations in liquid mixtures to measure the Soret and mass diffusion coefficient, J. Chem. Phys. 137, 234202 (2012).

[44] M. Italia, F. Croccolo, F. Scheffold, and A. Vailati, Inclined layer convection in a colloidal suspension with negative Soret coefficient at large solutal Rayleigh numbers, Eur. Phys. J. E 37, 101 (2014).

[45] G. S. Settles, Schlieren and Shadowgraph Techniques (Springer, Berlin, 2001).

[46] F. Croccolo and D. Brogioli, Quantitative Fourier analysis of schlieren masks: the transition from shadowgraph to schlieren, Appl. Opt. 50, 3419 (2011). 